\title{
The evolution process from silver nanoflakes to nanomeshes to effectively address the dilemma in the conductivity and stretchability trade-offs in elastic electrodes
}

\author{
Liqiao Chen, Zhe Leng, Yunqian Long, Xuan Yu, and Xiaoming Yu* \\ Institute of Innovation \& Application, Zhejiang Ocean University, Zhoushan 316022, P. R. China.
}

\begin{abstract}
Flexible and stretchable conductive materials have received significant attention in several applications such as flexible displays and sensors. In this paper, we report a highly dispersed porous Ag nanoflakes with clean surfaces were fabricated through an explosive growth process. The evolution process from silver nanoflakes to nanomeshes occurred by the novel "dissolution-recrystallization" solvothermal process. The as-obtained Ag meshes have the dual nature of nanoflakes and nanoparticles, which could create an intercross and interpenetration conductive network structures between silver and polymer in the printed elastic conductor, therefore, the silver meshes as conductive fillers used in elastic conductor simultaneously exhibit high conductivity and mechanical durability.
\end{abstract}

Keywords: Liquid phase transformation; Ag flakes; Ag meshes, elastic electrodes

\section{Introduction}

Flexible printed electronics aim to minimize material waste and production costs, leading to reduced carbon dioxide emissions during manufacturing. ${ }^{1,2}$ With the development of nanomaterials and nanotechnology, various kinds of printing pastes ranging from conductors, insulators and semiconductors comprised of nanostructures have been developed and utilized for solar cells ${ }^{3}$, touch screens, ${ }^{4}$ transistors,${ }^{5}$ sensors,${ }^{6,7}$ and elastic/flexible devices ${ }^{8,}{ }^{9}$ Flexibility and stretchable properties are the key parameters of flexible devices. ${ }^{10}$ The elastic electrodes are also necessary and important components for the future applications of flexible printed electronics.

Different micro/nano structures silver powders including Ag flakes, Ag meshes, Ag nanowires, and Ag nanoparticles were introduced to fabricate flexible electrodes. ${ }^{10,11}$ In spite of intensive efforts, two major issues still remain for currently existing stretchable conductive materials, that is, due to the trade-off of their mechanical and electrical characteristics, increasing the conductive fillers amount for 
higher conductivity increases the material stiffness which in turn diminishes the stretchability. ${ }^{2}$ Therefore, both the high conductivity and stretchability requires its conductive fillers (silver powders) for the flexible silver electrode preferably have the dual characteristics of flakes and nanoparticles. ${ }^{12,13}$ The different approaches have established to address the dilemma in the conductivity and stretchability trade-offs to some extent ${ }^{10,12}$.

Silver nanowires conductive network is a good choice to address the dilemma in the conductivity and stretchability trade-offs. However, to obtain good conductivity and stretchability, it is necessary to further treatment for silver nanowires network to form electrical and mechanical the junctions among the different nanowires. Welding the nanowire junctions is an effective strategy for reducing the sheet resistance and improving the operational stability of flexible nanowire electrode in practical applications. ${ }^{14,15}$ Chemically growing silver "solder" or the chemical treatment for the junctions of the nanowires can also contribute to low-cost and highly stable electrodes. ${ }^{16,17}$ Therefore, the formation and reliability of network junctions are the key factor to the performance of silver nanowire electrodes.

Here, we directly synthesized silver nanonets powders as conductive fillers of silver electrodes instead of silver nanowires conductive network. Firstly, we adopted a simple route to synthesize Ag porous nanoflakes composed of nanoparticles without using any capping agents. Interestingly, Ag nanoflakes could also convert into different morphologies such as irregular nanoparticles or porous meshes through the novel "dissolution-recrystallization" solvothermal process. We demonstrate the feasibility of nanoflakes as conducting fillers to fabricate elastic electrodes. Surprisingly, porous Ag meshes simultaneously exhibit high conductivity and mechanical durability. This is probably because the forming of the intercross and interpenetration structure between resins with Ag nanomeshes.

\section{Experimental}

Synthesis of typical silver nanoflakes. $\mathrm{AgNO}_{3}(8.0 \mathrm{mmol})$ was dissolved in $2.5 \mathrm{~mL}$ of water. $19.0 \mathrm{mmol}$ of L-ascorbic acid was dissolved in $2.5 \mathrm{~mL}$ ethanol and $5.0 \mathrm{~mL}$ water under vigorous magnetic stirring. Then, $0.1 \mathrm{mmol}$ of D-saccharic acid 1,4-lactone monohydrate was added into the ascorbic acid solution to prepare a reductive solution. Then, $\mathrm{AgNO}_{3}$ solution was quickly added into the reductive solution with vigorous stirring. After five minutes, a solid product was obtained, which was washed with distilled water and alcohol for several times.

Solvothermal transformation of typical silver flakes. First, the above samples were transferred to a 
Teflon-lined stainless-steel autoclave $(25 \mathrm{~mL})$ and sealed after adding $15.0 \mathrm{~mL} \mathrm{H}_{2} \mathrm{O}$. Solvothermal processing was carried out at $160^{\circ} \mathrm{C}$ for $2 \mathrm{~h}, 4 \mathrm{~h}, 6 \mathrm{~h}$, and $8 \mathrm{~h}$. The resulting products were washed with distilled water and alcohol several times. Finally, the product was dried at $60{ }^{\circ} \mathrm{C}$ for ca. $10 \mathrm{~h}$.

The products were characterized using powder XRD and scanning electron microscopy (SEM). XRD patterns were recorded using a Rigaku D/MAX 2200 VPC diffractometer using CuK $\alpha$ radiation $(\lambda=0.15045 \mathrm{~nm})$ and a graphite monochromator. SEM images were recorded using a FEI Quanta 400 Thermal FE environmental scanning electron microscope. TEM images were recorded using a JEM-2010HR transmission electron microscope operated at an accelerating voltage of $200 \mathrm{kV}$. TEM samples were prepared by dispersing the powders on a holey carbon film supported on copper grids.

The preparation and the evaluation of elastic conductors. The elastic conductor pastes are comprised of Ag flakes, polyester rubber and some other additives. First, polyester dissolved in the DBE solvent as adhesive agents, and then mixed the as-prepared silver powders (70 wt.\%), and processed into conductive paste. On the neoprene rubber sheet, a $15 \mathrm{~mm}$ width $160 \mathrm{~mm}$ length, and $1 \mathrm{~mm}$ thick coating plate was used. After screen printing a $5 \times 100 \mathrm{~mm}^{2}$ rectangular silver conductive film, the samples were dried at $150{ }^{\circ} \mathrm{C}$ for $30 \mathrm{~min}$. The sheet resistances of conductive films were measured for different bending cycle times and the rate of stretching length.

\section{Results and Discussion}

The powder X-ray diffraction patterns (XRD) of as typical synthesized sample are shown in Figure 1a. This matches well with JCPDS card no. 04-0783 for cubic Ag with $\mathrm{a}=\mathrm{b}=\mathrm{c}=0.4086 \mathrm{~nm}$, confirming that all the products were cubic-structure Ag. The SEM images of the samples (Figure 1b) show that Ag nanoflakes are with a width and thickness of approximately $10 \mu \mathrm{m}$ and $60 \mathrm{~nm}$, respectively, and dozens of nanometer-scale holes on its surface. The TEM images show those holes are irregular and the sizes are about tens of nanometers (Figure 1c). The structures of these nanoflakes are also conformed through HRTEM(Figure 1d).The lattice-resolved image taken on a single Ag nanoflakes reveals fringes with separated spacing of $0.25 \mathrm{~nm}$, being assigned to the $\mathrm{Ag} \quad(1 / 3)\{422\}$ reflection, which has also been previously observed for Ag nanoplates, the assignment is consistent with the geometrical model, in which each triangular nanoplate is bound by two $\{111\}$ planes as the top and bottom faces and three $\{100\}$ planes as the side faces. ${ }^{18}$ 

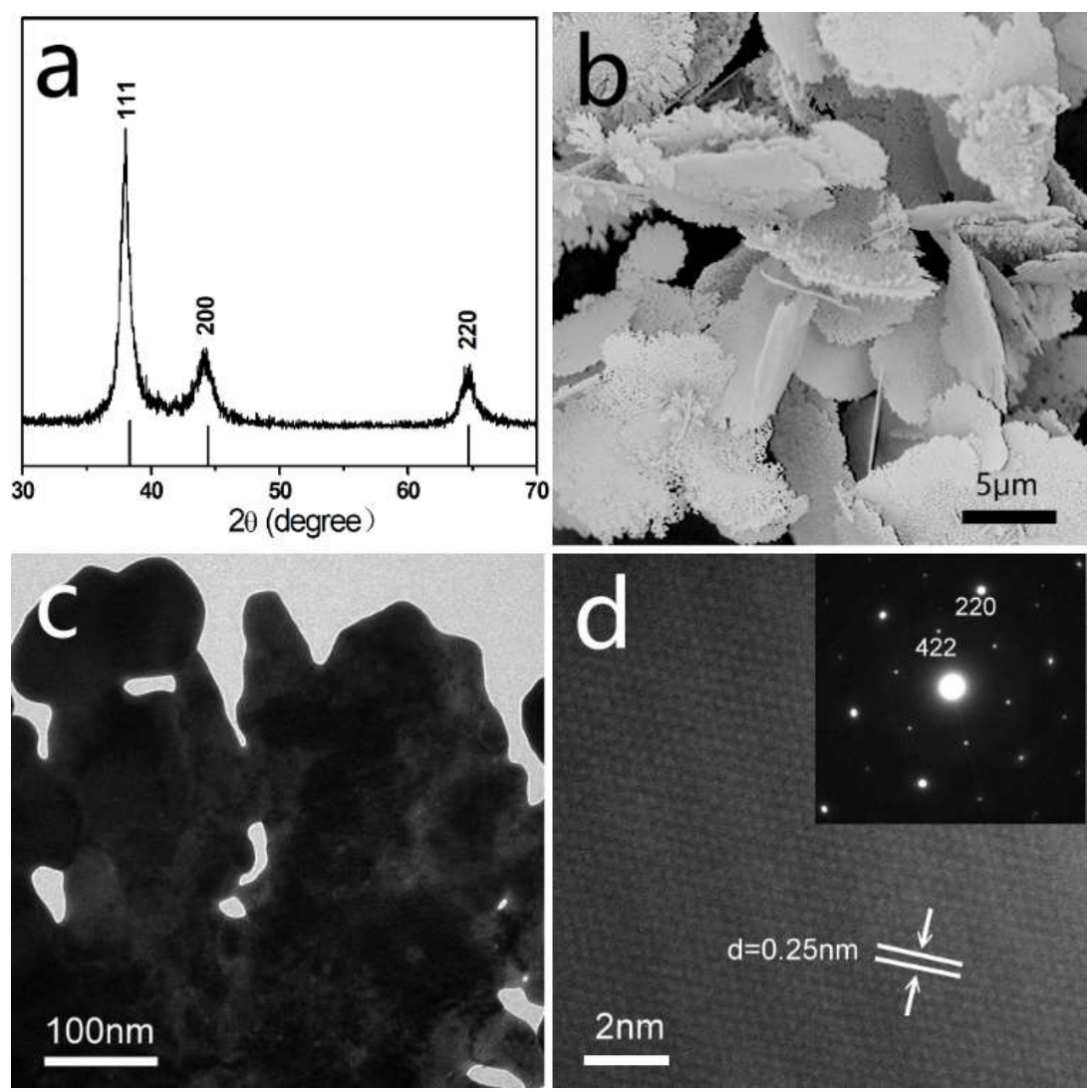

Figure 1. pXRD (a), SEM images (b) TEM images (c)HRTEM images and SEAD(inset) (d)of as-obtained Ag flakes.

Some nanostructures are unstable and they possibly changes under solvothermal condition as our previous reported. ${ }^{19}$ Thus, we investigated the evolution of the structure of $\mathrm{Ag}$ nanoflakes under different solvothermal processing. The as-obtained Ag nanoflakes were differently added to $10 \mathrm{~mL}$ of distilled water, $0.01 \mathrm{mM} \mathrm{HCL} /$ water, $0.01 \mathrm{mM} \mathrm{NaOH} /$ water, and diethylene glycol aging at $150{ }^{\circ} \mathrm{C}$ for 6h. As shown in the SEM images in Figures $2 \mathrm{a}-2 \mathrm{~d}$, the silver powders become irregular shape, and the sizes of nanoflakes decrease, and their thickness increase after aging in pure water for $6 \mathrm{~h}$. In the condition of $\mathrm{pH}=4$, the as synthesized products are an irregular and spherical shapes. When increase the $\mathrm{pH}$ of reaction solution to alkaline $(\mathrm{pH}=10)$, some irregular shape with smaller particle sizes could be obtained. The sizes of Ag flakes are smaller and thicker, and the sizes of the cores in nanoflakes increase when diethylene glycol was used instead of water. 


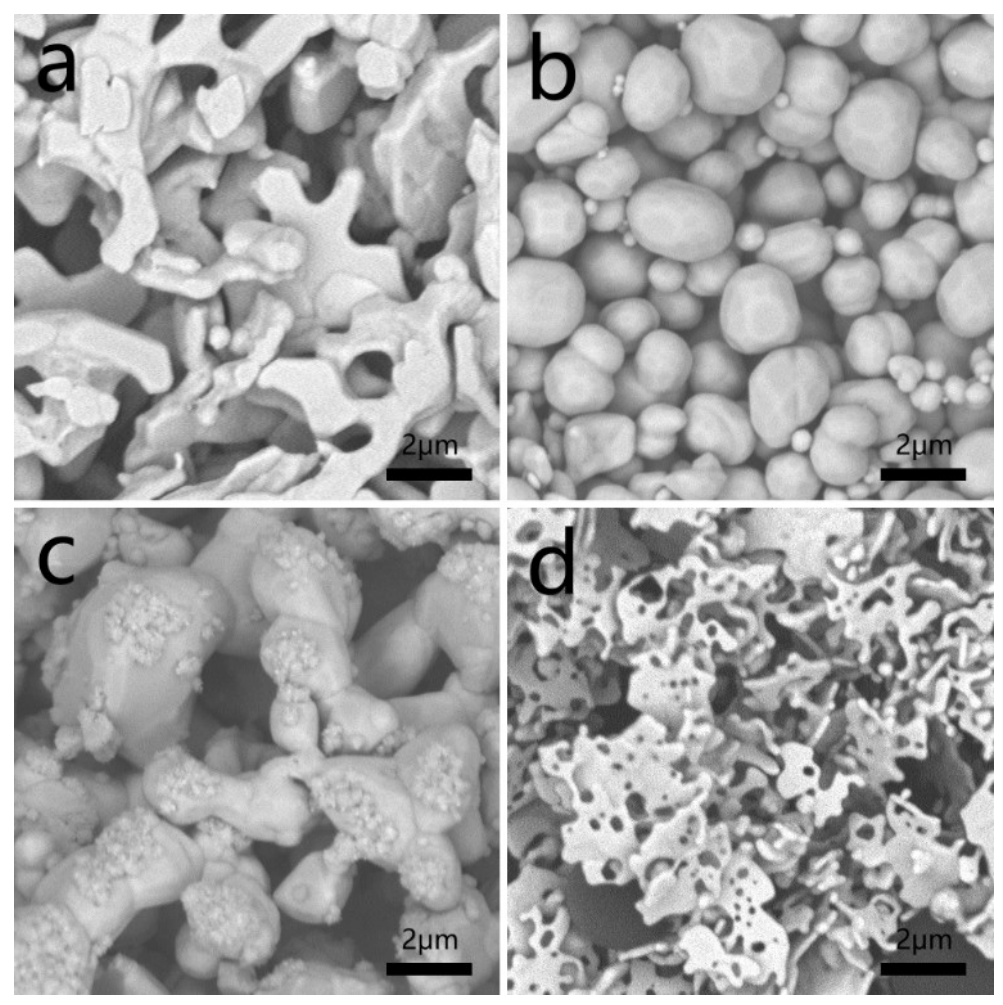

Figure 2. SEM images of the sample obtained after aging at $150^{\circ} \mathrm{C}$ for $6 \mathrm{~h}$ in DI water (a); $0.01 \mathrm{mM}$ $\mathrm{HCl} / \mathrm{DI}$ water solution (b); 0.01mM NaOH/DI water solution (c); diethylene glycol (d).

In our previous paper, we reported that $\alpha-\mathrm{Fe}_{2} \mathrm{O}_{3}$ flakes could gradually transformed into nanoparticles through "dissolution-recrystallization" solvothermal process and "a mass transport" took place $^{19}$. Herein, the experiments showed that Ag nanoflakes have similar features. Further study on the structural evolution mechanism of Ag nanoflakes might help to better understand the physical process by monitoring the evolution of Ag flakes at different times.

The as-synthesized Ag nanoflakes were added to $10 \mathrm{~mL}$ of distilled water. The growth process of this route was continuously monitored by collecting the samples from different aging times. The morphology was characterized by SEM (Figure 3). As shown in Figure 3a, the density of holes in the Ag flakes decrease, and the diameters of holes increase from 100 to $200 \mathrm{~nm}$ when the particles aged for $0.5 \mathrm{~h}$, also, the thickness of flakes increase. If prolonging the reaction time to $4 \mathrm{~h}$, the sizes of the flakes and the number of holes decrease (Figure 3b). By increasing the aging time to $8 \mathrm{~h}$, the flake-like structures almost disappear, and irregular shapes like biscuits are obtained (Figure 3c). All the holes vanish, and the sizes of the products reduce to less than $2 \mu \mathrm{m}$ when the aging time is extended to $24 \mathrm{~h}$. 


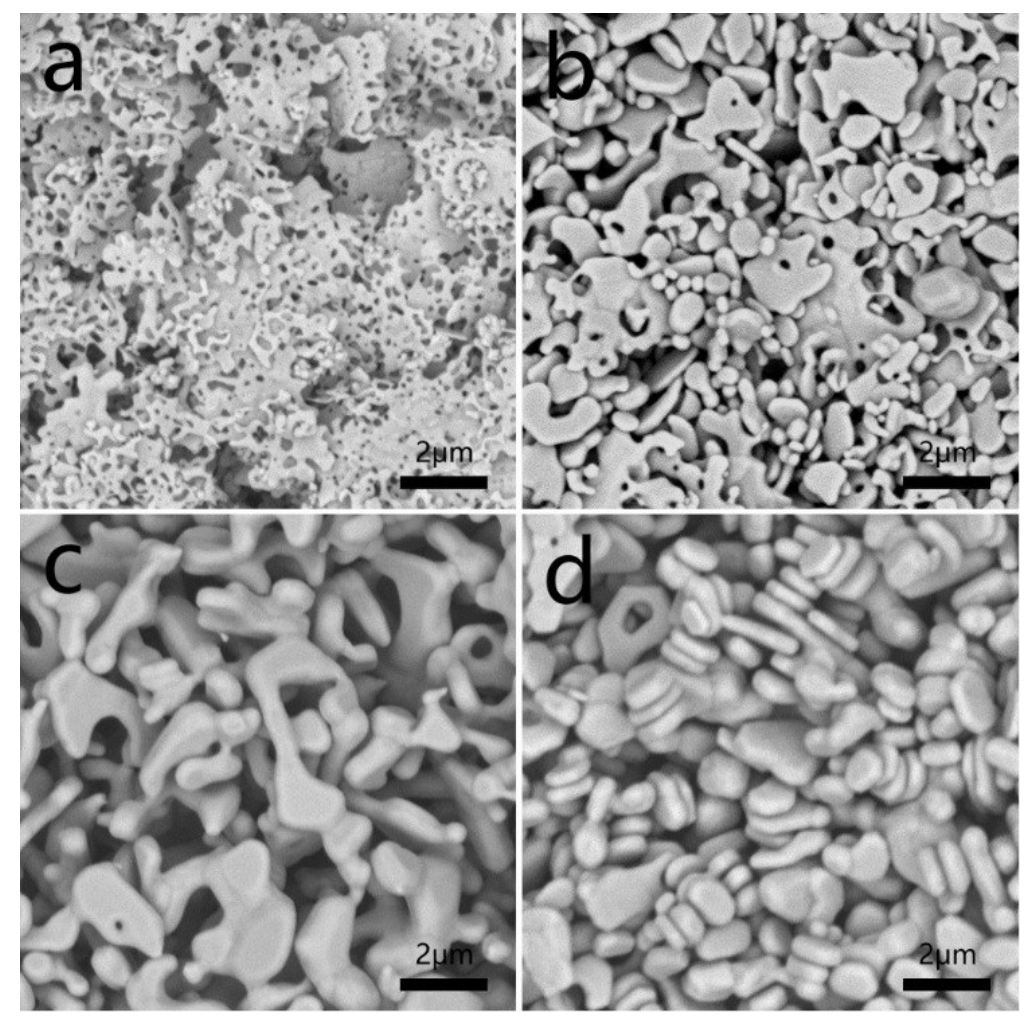

Figure 3. SEM images of Ag flakes evolution after aging for $0.5 \mathrm{~h} \mathrm{(a),} 4 \mathrm{~h} \mathrm{(b),} 8 \mathrm{~h}$ (c), and 24h (d) at $150{ }^{\circ} \mathrm{C}$.

The evolution processes of Ag nanostructures could be illustrated in Figure 4. First, the edges of Ag flakes and their holes gradually dissolve, so the sizes of holes become larger, and the diameter of flakes decrease. According to "Ostwald ripping" mechanism, ${ }^{20}$ that is, the small particles gradually dissolve due to the high surface energy, and the large particles continue to grow. Herein, small holes gradually disappear, and larger holes gradually break into a few particles. Continuous mass transport and growth from two dimensions to the three dimensions ultimately evolve into a stable structure with a low surface energy. ${ }^{19}$ 


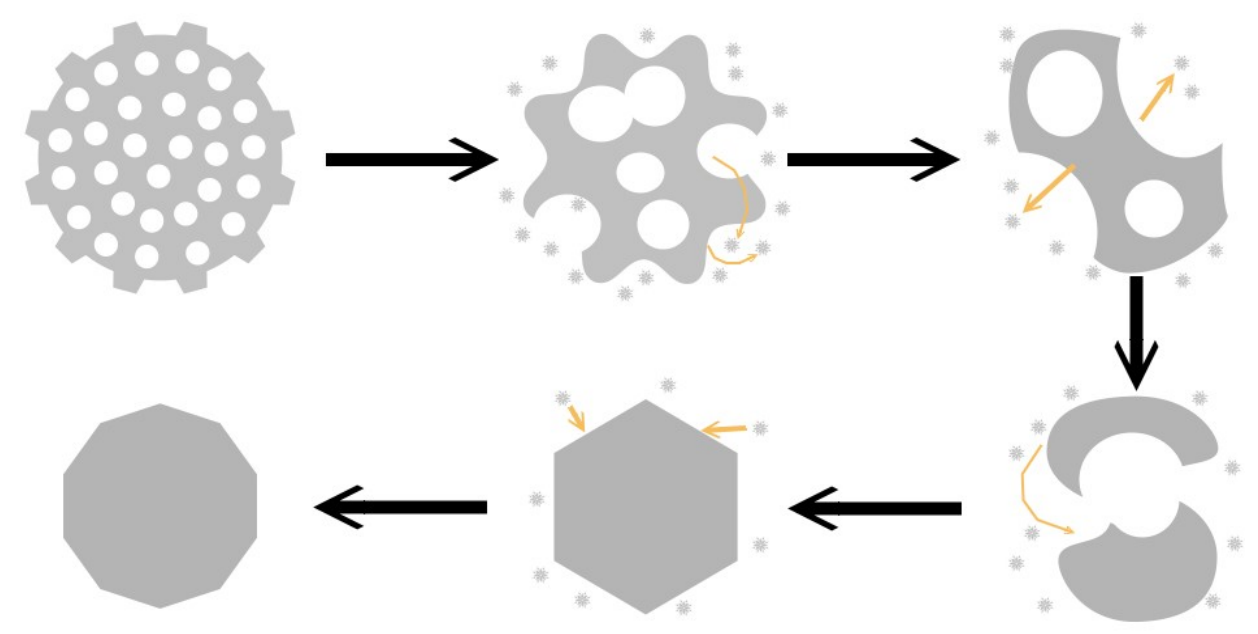

Figure 4. Schematic diagram of the structure evolution from the surface-roughened Ag flakes to $\mathrm{Ag}$ porous mesh.

Further, we prepared four conductive pastes using four kinds of silver powders in figure 3as conductive fillers and compared their flexibility of the four samples, respectively. The four conductors were obtained when the four conductive pastes were printed were heated for $30 \mathrm{~min}$ and named for sample $1 \#$, sample 2\#, sample 3\#, sample 4\#.According to the results showed in Figure 5 (a), the resistance of four samples all increased when the conductors were stretched, but the increasing value of sample $1 \#$ was obviously smaller than that of the other three samples. For example, the stretching rate of conductor for sample $1 \#$ was $140 \%$, and the resistance increased by $3500 \%$, but sample $4 \#$ lost conductive function at the same stretching rate. Therefore, the silver powders with more uniform, mesh-structure significantly improves the stretching properties of the conductor. The resistance changes of the four samples after bending for multiple cycles are also measured. The results are shown in figure 5 (b). Similar, the sample $1 \#$ showed more excellent anti-bending conductivity than the other samples. Although the anti-bending conductivity of all four samples are still not very satisfied, owe to the complexity of conductor system, such as the selection of rubbers and other agents are also critical and their compatibility with the surfactant, ${ }^{1}$ much efforts and optimized experiments would be necessary and going on. 

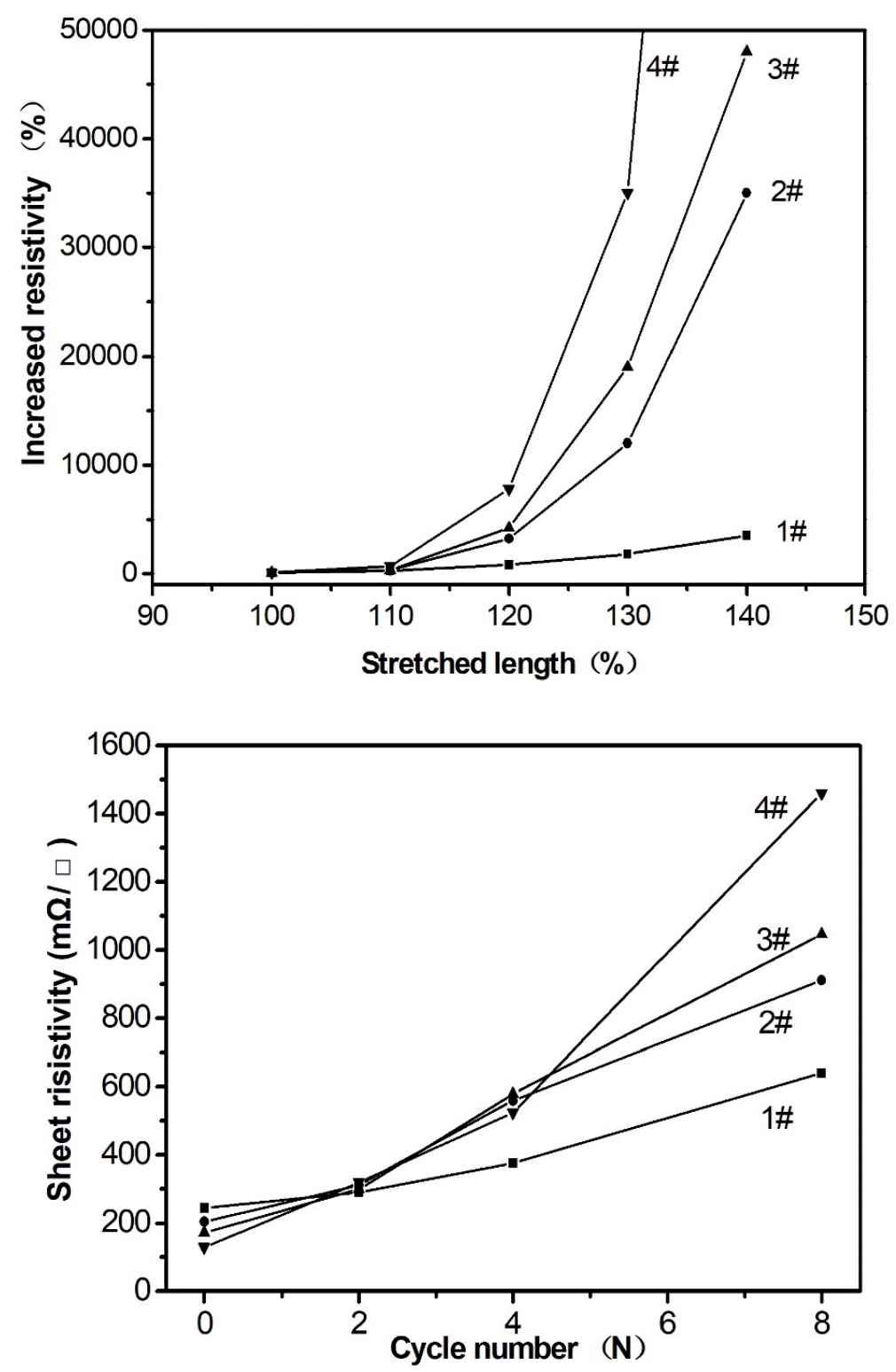

Figure 5. Surface resistance of samples1\#, 2\#, 3\#, 4\# changes with bending cycle times (a)and the rate of stretching length (b).

The reason that enhanced conductivity and stretchability are simultaneously realized by the introduction of the nanomeshes as conductive fillers can be attributed to their special structures. The deformation of conductive networks during stretching or bending could destroy the conductive channel in conductors. The schematic diagram was shown in Figure 6. When Ag meshes were used as the conductive fillers, Ag meshes are uniformly dispersed in a rubber matrix, and the Ag meshes could form an intercross and interpenetration structure with resin where resin or rubber molecular are 
connected with nanomeshes through via-holes to maintain mechanical durability, at the same time, conductive paths in silver conductive networks would have few influences after bending or stretching. The combination of conductivity and stretchability are enabled by a special conductive network structure of intercross and interpenetration between silver and polymer matrix in the printed elastic conductor. ${ }^{21}$ For the case of nanoparticles or nanoflakes, the contact point or contact area reduce when stretching happen, which resulted in rapid increase of resistance.

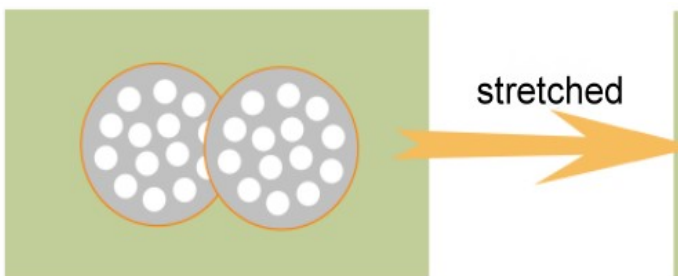

Silver nets

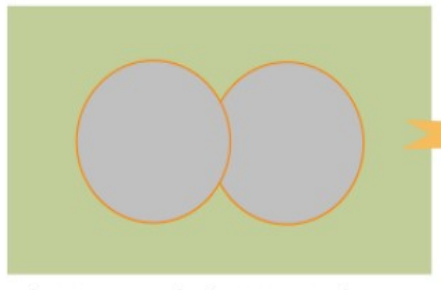

Ag nanoplates or spheres

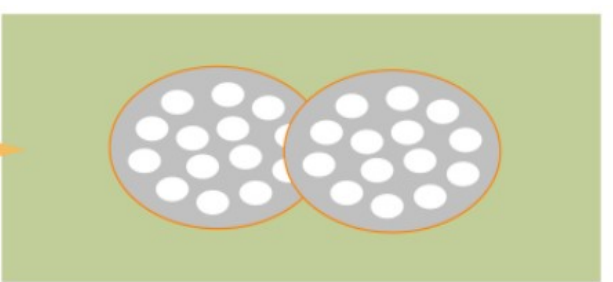

Silver nets distortion

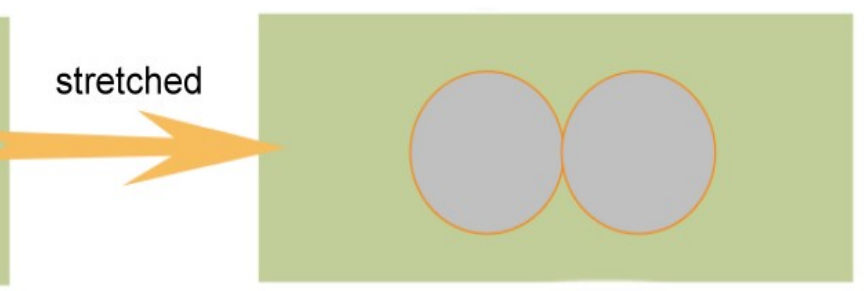

Ag particles separation

Figure 6. The schematic diagram for the change of contact areas in conductors with different forms of silver fillers when stretching happen.

\section{Conclusion}

In conclusion, we adopted a simple route to investigate the transformation process from flakes to a porous mesh using "dissolution-recrystallization" process. The reaction system in this method is simple, and the samples obtained were dispersed with a clean surface because of only water was added without adding any other surfactants or reagents. This route not only provided the convenience for novel Ag nanostructures, but also offered a special conductive filler to realize elastic conductors, which verified to be new electronic functional fillers that simultaneously exhibit high conductivity and mechanical durability in elastic electrodes. 


\section{Acknowledgements}

This work was supported from the National Natural Science Foundation of China (Grant No.51261008, 11604298,51501046) and Scientific Research Foundation (Q1444) of Zhejiang Ocean University.

\section{References}

1. Matsuhisa, N.; Kaltenbrunner, M.; Yokota, T.; Jinno, H.; Kuribara, K.; Sekitani, T.; Someya, T. Nature Communications 2015, 6, 7461.

2. Wang, J.; Cai, G.; Li, S.; Gao, D.; Xiong, J.; Lee, P. S. Advanced Materials 2018, 30, (16), 1706157.

3. Hu, Y.; Zhang, Z.; Mei, A.; Jiang, Y.; Hou, X.; Wang, Q.; Du, K.; Rong, Y.; Zhou, Y.; Xu, G. Advanced Materials 2018, 30, (11), 1705786.

4. Guzman, N. D.; Jr, M. R.; Balela, M. D. Materials Research Bulletin 2018, 106, 446-454.

5. Tang, W.; Huang, Y.; Han, L.; Liu, R.; Su, Y.; Guo, X.; Yan, F. Journal of Materials Chemistry C 2019, 7 .

6. $\quad$ Ma, Z.; Ping, C.; Wen, C.; Yan, K.; Pan, L.; Shi, Y.; Yu, G. Nano Letters 2018, 18, (7), 4570-4575.

7. Foo, C. Y.; Lim, H. N.; Mahdi, M. A.; Wahid, M. H.; Huang, N. M. Scientific Reports 2018, 8, (1).

8. Ko, G. J.; Shin, J. W.; Hwang, S. W., Flexible/Stretchable Devices for Medical Applications. 2018.

9. Shinde, S. M.; Das, T.; Hoang, A. T.; Sharma, B. K.; Xiang, C.; Ahn, J. H. Advanced Functional Materials 2018.

10. Wang, Z.; Wei, W.; Jiang, Z.; Dan, Y. Progress in Organic Coatings 2016, 101, 604-611.

11. Shinde, M. A.; Mallikarjuna, K.; Noh, J.; Kim, H. Thin Solid Films 2018, 660, 447-454.

12. Matsuhisa, N.; Inoue, D.; Zalar, P.; Jin, H.; Matsuba, Y.; Itoh, A.; Yokota, T.; Hashizume, D.; Someya, T. Nature Materials 2017, 16, (8), 834.

13. Ruo-Zhou, L.; Anming, H.; Denzel, B.; Tong, Z.; Oakes, K. D.; Rui, P.; Uma, T.; Zili, W.; Zhili, F. Nanoscale 2015, 7, (16), 7368-7377.

14. Garnett, E. C.; Wenshan, C.; Cha, J. J.; Fakhruddin, M.; Connor, S. T.; Christoforo, M., Greyson; Yi, C.; Mcgehee, M. D.; Brongersma, M. L. Nature Materials 2012, 11, (3), 241.

15. Park, J. H.; Hwang, G. T.; Kim, S.; Seo, J.; Park, H. J.; Yu, K.; Kim, T. S.; Lee, K. J. Advanced 
Materials 2017, 29, (5), -.

16. Lu, H.; Zhang, D.; Cheng, J.; Liu, J.; Mao, J.; Choy, W. C. H. Advanced Functional Materials 2015, 25, (27), 4211-4218.

17. Kim, S.; Jeon, H. R.; An, C.-H.; An, B.-S.; Yang, C.-W.; Lee, H.-J.; Weon, B. M. Materials Letters 2017, 193, 63-66.

18. Washio, I.; Xiong, Y.; Yin, Y.; Xia, Y. Advanced Materials 2010, 18, (13), 1745-1749.

19. Chen, L.; Xiong, Q.; Li, W.; Li, J.; Yu, X. CrystEngComm 2015, 17, (45), 8602-8606.

20. Dennis, N. W.; Muhoberac, B. B.; Newton, J. C.; Kumbhar, A.; Sardar, R. Plasmonics 2014, 9, (1), 111-120.

21. Lee, H. B.; Jin, W. Y.; Ovhal, M. M.; Kumar, N.; Kang, J. W. Journal of Materials Chemistry C $2018,7$. 